\title{
Annotation of expressed sequence tags for the East African cichlid fish Astatotilapia burtoni and evolutionary analyses of cichlid ORFs Walter Salzburger $^{\dagger 1,2}$, Susan CP Renn ${ }^{\dagger 3}$, Dirk Steinke ${ }^{\dagger 1,4}$, Ingo Braasch ${ }^{1,5}$, Hans A Hofmann ${ }^{6}$ and Axel Meyer*1
}

\begin{abstract}
Address: ${ }^{1}$ Lehrstuhl für Zoologie und Evolutionsbiologie, Department of Biology, University of Konstanz, 78467 Konstanz, Germany, ${ }^{2 Z}$ Zoological Institute, University of Basel, 4051, Switzerland, ${ }^{3}$ Department of Biology, Reed College, Portland, Oregon 97202, USA, ${ }^{4}$ Guelph Centre for DNA Barcoding, Biodiversity Institute of Ontario, University of Guelph, Guelph, Ontario N1G 2W1, Canada, ${ }^{5}$ Physiological Chemistry I, Biozentrum, University of Würzburg, 97074 Würzburg, Germany and 'Section of Integrative Biology, University of Texas at Austin, Austin, Texas 78712, USA

Email: Walter Salzburger - walter.salzburger@unibas.ch; Susan CP Renn - renns@reed.edu; Dirk Steinke - dsteinke@uoguelph.ca; Ingo Braasch - ingo.braasch@biozentrum.uni-wuerzburg.de; Hans A Hofmann - hans@mail.utexas.edu; Axel Meyer* - axel.meyer@unikonstanz.de

* Corresponding author †Equal contributors
\end{abstract}

Published: 25 February 2008

BMC Genomics 2008, 9:96 doi:10.1 186/147|-2164-9-96
Received: II October 2007

Accepted: 25 February 2008

This article is available from: http://www.biomedcentral.com/I47I-2/64/9/96

(c) 2008 Salzburger et al; licensee BioMed Central Ltd.

This is an Open Access article distributed under the terms of the Creative Commons Attribution License (http://creativecommons.org/licenses/by/2.0), which permits unrestricted use, distribution, and reproduction in any medium, provided the original work is properly cited.

\begin{abstract}
Background: The cichlid fishes in general, and the exceptionally diverse East African haplochromine cichlids in particular, are famous examples of adaptive radiation and explosive speciation. Here we report the collection and annotation of more than 12,000 expressed sequence tags (ESTs) generated from three different CDNA libraries obtained from the East African haplochromine cichlid species Astatotilapia burtoni and Metriaclima zebra.

Results: We first annotated more than 12,000 newly generated cichlid ESTs using the Gene Ontology classification system. For evolutionary analyses, we combined these ESTs with all available sequence data for haplochromine cichlids, which resulted in a total of more than 45,000 ESTs. The ESTs represent a broad range of molecular functions and biological processes. We compared the haplochromine ESTs to sequence data from those available for other fish model systems such as pufferfish (Takifugu rubripes and Tetraodon nigroviridis), trout, and zebrafish. We characterized genes that show a faster or slower rate of base substitutions in haplochromine cichlids compared to other fish species, as this is indicative of a relaxed or reinforced selection regime. Four of these genes showed the signature of positive selection as revealed by calculating $\mathrm{K}_{\mathrm{a}} / \mathrm{K}_{\mathrm{s}}$ ratios.

Conclusion: About $22 \%$ of the surveyed ESTs were found to have cichlid specific rate differences suggesting that these genes might play a role in lineage specific characteristics of cichlids. We also conclude that the four genes with a $\mathrm{K}_{\mathrm{a}} / \mathrm{K}_{\mathrm{s}}$ ratio greater than one appear as good candidate genes for further work on the genetic basis of evolutionary success of haplochromine cichlid fishes.
\end{abstract}

\section{Background}

The exceptionally diverse species flocks of cichlid fishes in the East African Great Lakes Tanganyika, Malawi and Victoria are prime examples for adaptive radiations and explosive speciation [1-3]. More than 2,000 cichlid species have evolved in the last few million years in the rivers and lakes of East Africa [1,4-6]. Together with an additional $\sim 1,000$ species that are found in other parts of 
Africa, in South and Central America, in Madagascar, and in India, the family Cichlidae represents one of the most species-rich families of vertebrates. In addition to their unparalleled species-richness, cichlids are famous for their ecological, morphological and behavioral diversity $[1,2,7]$, for their propensity for rapid speciation [5], for their capacity for sympatric speciation $[8,9]$, and for the formation of parallel characters in independently evolved species flocks [10-12]. For these reasons, the cichlid fishes are an excellent model system to study basic dynamics of evolution, adaptation and speciation. However, while the phylogenetic relationships between the main cichlid lineages are largely established and some of the cichlids' evolutionary innovations have been identified $[1,2,4,7,13]$, little is known about the genomic and transcriptional basis of the evolutionary success of the cichlids.

The cichlid model system provides many advantages for evolutionary genomic research. The hundreds of closely related yet morphologically diverse species in East Africa's cichlid species flocks are even more powerful than a 'mutagenic screen' (to which these species assemblages have been compared $[1,12]$ ) in that they represent combinations of alleles that confer a selective advantage under various ecological pressures. Because of the possibility to produce viable crosses between different cichlid species in the lab [14], these alleles can be tied to particular phenotypic traits by means of classical genetic experiments [1518]. The close relatedness of the different species allows the design of primer sets for the amplification of particular genomic DNA regions such as candidate gene loci, microsatellites, or SNPs, which are applicable to a wide range of species $[17,19-21]$. The same is true for expression profiling with CDNA microarrays that, once developed for one species, can be used for any East African cichlid species [22].

A variety of genomic resources have already been established for East African cichlid species. Genetic maps are available for the Nile tilapia Oreochromis niloticus $[23,24]$ and the Lake Malawi species Metriaclima zebra [17]. BAC libraries have been constructed for O. niloticus [25] and $M$. zebra (available at the Hubbard Center for Genome Studies), for the Lake Victoria haplochromine Paralabidochromis chilotes [26] and for Astatotilapia burtoni from Lake Tanganyika and surrounding rivers [27]. cDNA microarrays are available for A. burtoni [22] and for Lake Victoria haplochromines $[28,29]$. Also, EST sequencing projects have been initiated [30], and a BLAST server for cichlid resources has been established [31]. Recently, the National Institute of Health (NIH) has committed to sequencing four cichlid genomes. A detailed description of genomic resources developed for cichlid fishes is available at [32].
Expressed sequence tags (ESTs) derived from the partial sequencing of cDNA clones provide an economical approach to identify large numbers of genes that can be used for comparative genomic and gene expression studies as well as for the detection of splice variants $[33,34]$. Furthermore, EST projects facilitate genome annotation and are therefore often applied in addition to genome sequencing projects. Due to the large amount of data available in public databases, ESTs emerge as important resources for comparative genome-wide surveys both among closely and more distantly related taxa $[35,36]$. A series of software applications have been developed to date to perform such EST-based analyses [37-39]. Since ESTs reflect the coding portions of a genome, they can also be used to test for different evolutionary rates in particular genes when comparing different lineages, and to detect genes that have undergone positive selection [35]. It is generally assumed that genes with a statistically significant increase in substitution rates have experienced relaxed functional constraints, while genes, which have not undergone accelerated substitution rates, have experienced purifying selection and, thus, could not accumulate substitutions at random. Positive Darwinian selection, on the other hand, is a phenomenon where selective pressure is favoring change. Natural selection is commonly thought of as a process of editing genetic change so that only a small number of mutational events are retained in natural populations. Under positive selection, the retention of mutations is much closer to the rate at which mutations occur.

Here we report the collection and annotation of more than 12,000 ESTs generated from two different cDNA libraries obtained from the East African cichlid species Astatotilapia burtoni, as well as a smaller cDNA library from the Lake Malawi species Metriaclima zebra. Astatotilapia burtoni has long been used as a model system to study cichlid spawning behavior $[7,40,41]$, social interactions [41-44], neural and behavioral plasticity $[45,46]$, endocrinology [47], the visual system [48], as well as cichlid development and embryogenesis [49]. In addition, the phylogenetic position of $A$. burtoni makes this species an ideal model system for comparative genomic research [27]. Astatotilapia burtoni, which belongs to the most species-rich lineage of cichlids, the haplochromines, was shown to be a sister group to both the Lake Victoria region superflock ( 600 species) and the species flock of Lake Malawi ( 1,000 species) $[4,5,50,51]$. Three highly specialized haplochromine species from two species assemblages, Paralabidochromis chilotes and Ptyochromis sp. "redtail sheller" from Lake Victoria and Metriaclima zebra from Lake Malawi, have already been established as genomic models $[16,26,28,30]$. Important insight into cichlid (genome) evolution will be afforded by the comparison of their genomes to that of A. burtoni, which has a 
more generalist life style and is likely to resemble the ancestral lineage that seeded the cichlid adaptive radiations in these two lakes $[4,7]$.

For EST sequencing, we utilized a cDNA library from $A$. burtoni brain tissue ('brain') that was used for the construction of a cDNA microarray [22] and a newly generated normalized cDNA library constructed from different $A$. burtoni tissues at different developmental stages ('pinky'). We annotated the ESTs on the basis of similarity searches with BLAST and using the structured vocabulary provided by the Gene Ontology Consortium [52], based on molecular studies of gene function in various model organisms [53]. For evolutionary analyses, we combined our newly generated ESTs with all available sequence data for haplochromine cichlids [30] and a previously constructed library from skin tissue of the Lake Malawi species Metriaclima zebra (W. Salzburger, H. A. Hofmann \& A. Meyer; unpublished data), which resulted in a total of more than 45,000 ESTs. We then compared the haplochromine ESTs to sequence data from two pufferfish species (Takifugu rubripes and Tetraodon nigroviridis), trout, and zebrafish, and identified those ESTs with cichlid specific differences in evolutionary rates with EverEST [37].

\section{Results}

The 14,592 initial sequences were trimmed of vector and low-quality sequences and filtered for minimum length (200 bp cut-off), identifying 12,070 high-quality ESTs (Table 1). More than 11,000 of these ESTs (from 13,056 initial sequences) are derived from two different Astatotilapia burtoni cDNA libraries - one made from brain tissue ('brain'), the other one from different tissues ('pinky') including brain, muscle, skin and fin. The overall quality as measured by sequencing success rate and read-length was better in the 'pinky' library. Also, there was much less redundancy in the 'pinky' library (16\% versus 30\%), which might be the consequence of the normalization step applied to this library or the use of different source tissues.

A total of 8,636 A. burtoni sequences assembled into EST contigs have an open reading frame (ORF) of at least 400 bp. Of these, 1,219 (14\%) had matches in the Takifugu database and 7,417 (86\%) had no matches when an expected value threshold (e-value) of $<1 \times 10^{-50}$ was used. 2,902 (34\%) had matches in the Takifugu database with an expected value threshold of $<1 \times 10^{-15}$ and 3,460
(40\%) had matches with an expected value of $<1 \times 10^{-5}$. Similar proportions were retrieved with other databases (Fig. 1).

Among the 8,363 A. burtoni assembled sequences, 2,977 could be annotated according to Gene Ontology (GO) terms. Additional files 1, 2 and 3 use the generic GO slim subset of terms ([54]; Generic GO slim; Mundodi and Ireland; downloaded 04/06/2007) that have been developed to provide a useful summary of GO annotation for comparison of genomes, microarrays, or cDNA collections when a broad overview of the ontology content is required. 2,692 ESTs could be assigned to genes listed in the molecular function ontology, 2,532 to genes listed in the biological process ontology, and 2,293 to genes listed in the cellular components ontology, when using an evalue of $<1 \times 10^{-12}$. Additional files 4, 5, and 6 provide more detail of the specific fine-grained terms. Because a single A. burtoni assembled sequence may be annotated in all three ontologies and according to multiple ontology terms, a total of 27,451 annotations have been applied (10,926 among biological process, 9,414 among molecular function, and 7,111 among cellular component).

For the comparative evolutionary analyses, we combined our newly generated ESTs with previously published data from Paralabidochromis chilotes and P. sp. "redtail sheller" [30] and about 1,000 sequences obtained from a Metriaclima zebra skin cDNA library (W. Salzburger, H. A. Hofmann \& A. Meyer; unpublished data). When using this set of haplochromine cichlid ESTs as reference, we identified 759 open reading frames that are present in all six databases used for comparative analyses (haplochromine cichlids, Danio rerio, Homo sapiens, Oncorhynchus mykiss, Takifugu rubripes, and Tetraodon nigroviridis).

In order to identify sequences that evolve significantly more rapidly or more slowly in the haplochromine cichlid, we applied the triangle method implemented in EverEST [37] to calculate the p-distance for each of these 759 ORFs in all fish species relative to the human ortholog. There were 22 cases in which more than one haplochromine sequence was found. In these cases, we used the longest sequence for further analyses. The relative p-distances for three fish species were then mapped in ternary diagrams. An example of such a ternary diagram is shown

Table I: Expressed sequence tag (EST) summary

\begin{tabular}{lc}
\hline Total sequences & 13,056 \\
High quality sequences & 12,070 (between 200 and I,564 bP) \\
Brain library (A. burtoni) ('brain') & 4,570 \\
Mixed tissue library (A. burtoni) ('pinky') & 6,541 \\
Skin library (P. zebra) & 959
\end{tabular}




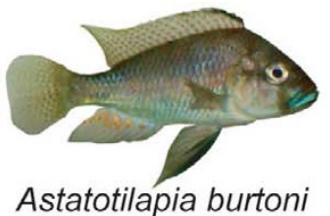

Astatotilapia burtoni

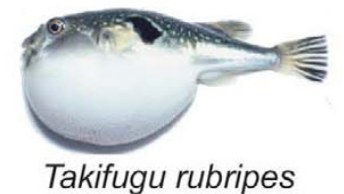

Takifugu rubripes

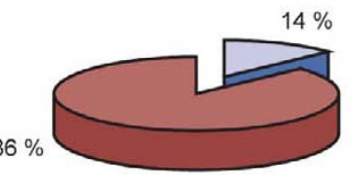

e-value $<10^{-15}$

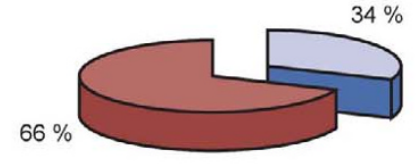

e-value $<10^{-5}$

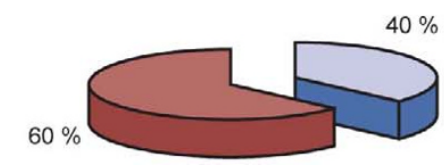

e-value $<10^{-50}$

$60 \%$

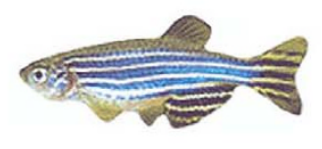

Danio rerio
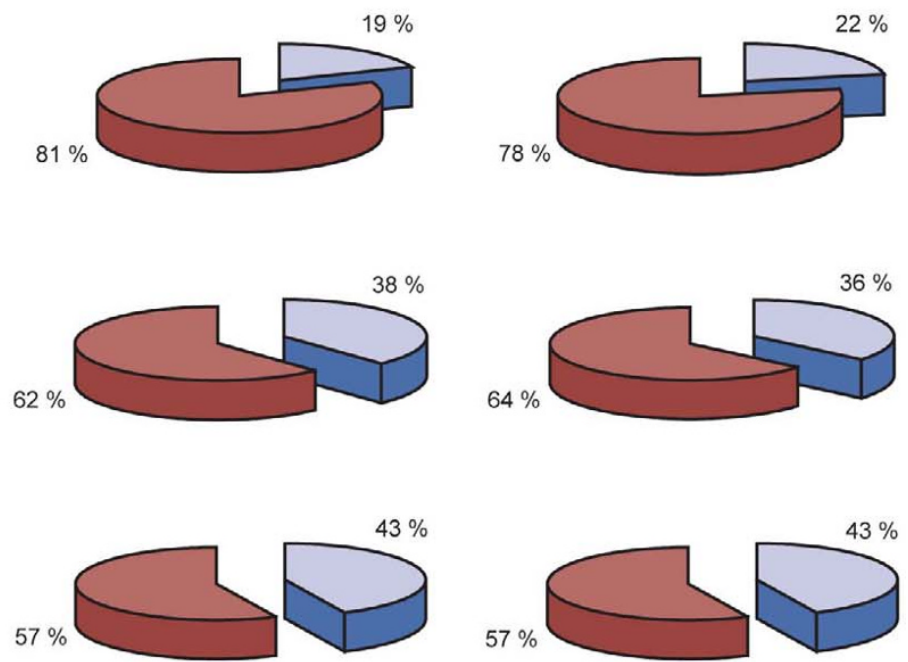

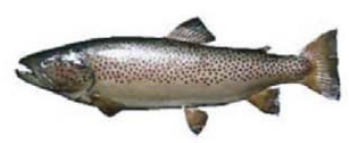

Oncorhynchus mykiss
$57 \%$$$
\text { . }
$$

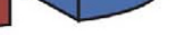

\section{Figure I}

The proportion of assembled haplochromine cichlid sequences with and without BLAST matches compared to three databases (Takifugu rubripes, Danio rerio, and Oncorhynchus mykiss). The pie charts indicate the relative number of BLAST hits (blue) versus the percentage fraction, for which no BLAST hit was retrieved (red) for three different evalues $\left(<10^{-50},<10^{-15}\right.$, and $<10^{-5}$, respectively).

in Fig. 2a, in this case showing the relative p-distances of cichlid, Takifugu rubripes, and Danio rerio amino acid sequences with respect to the homologous Homo sapiens genes. Figure 2b depicts a diagram with Oncorhynchus mykiss amino acid sequence divergence instead of haplochromine cichlid. The ternary diagrams show that in all combinations most genes are clustered around the center of the respective triangle, which indicates that, in general, the p-distances relative to the human outgroup are similar in all fish species.

When compared to the green-spotted pufferfish (Tetraodon nigroviridis) and fugu (Takifugu rubripes) (always with human as outgroup), 49 gene fragments appeared to have a significantly faster rate of evolution in haplochromine cichlids, and 213 had a slower rate. In the comparison including zebrafish and fugu, 52 genes were found to have evolved faster and 185 genes slower in cichlids. When trout and zebrafish were used, 69 genes were faster and 139 genes evolved slower. In a comparison including trout and fugu, 68 genes appeared to have a faster rate in haplochromines, and 132 had a slower rate. In total 69 genes were found to have evolved faster, and 213 genes appeared to have evolved with a significantly slower mutation rate in haplochromines compared to other fish species. Altogether, about $22 \%$ of the surveyed ESTs were found to have haplochromine specific rate differences in at least one of the comparisons suggesting that these genes might play a role in lineage specific features of haplochromine cichlids. A set of 170 cichlid genes appeared in all comparisons. Forty-eight cichlid genes were found to have a higher rate of amino-acid substitution compared to the other fish species included in this study, while 122 cichlid genes were found to have a slower rate. Cichlid sequences that match Danio rerio, Takifugu rubripes, Tetraodon nigroviridis, and Oncorhynchus mykiss genes and have a significantly higher or lower p-distance compared to the other fish genes relative to the human outgroup are listed in Additional files 7 and 8, respectively.

A histogram of the abundance of amino acid sequence divergences of all five fish species with respect to homologous human genes is depicted in Fig. 3. The p-distances appear normally distributed. With 0.211 , cichlids show the lowest average distance followed by Oncorhynchus mykiss (0.216), Danio rerio (0.239), Takifugu rubripes (0.242), and Tetraodon nigroviridis (0.258). The average distance of all five fish species to Homo sapiens is 0.233 . 

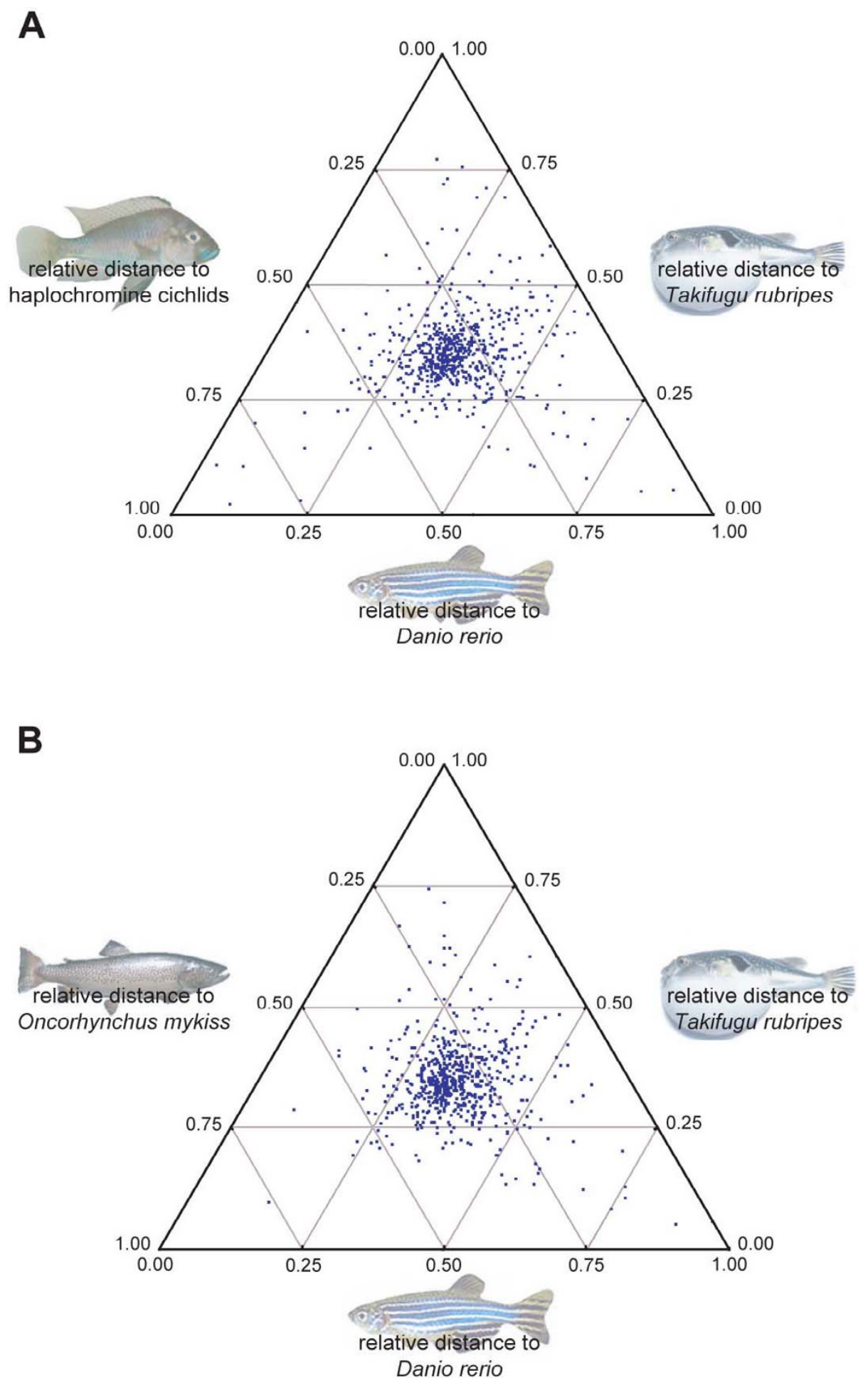

Figure 2

Ternary representation of relative distances of ORFs of three fish species compared to their human orthologs. (a) Haplochromine cichlid, Danio rerio, and Takifugu rubripes, (b) Danio rerio, Oncorhynchus mykiss, and Takifugu rubripes. Each dot represents a single ORF, the position of the dot within the ternary diagram indicates the relative distance of this ORF in each of the three fish species compared to the orthologous ORF in human. We were interested in identifying those ORFs that show a faster or slower rate of molecular evolution in the haplochromine cichlids. 


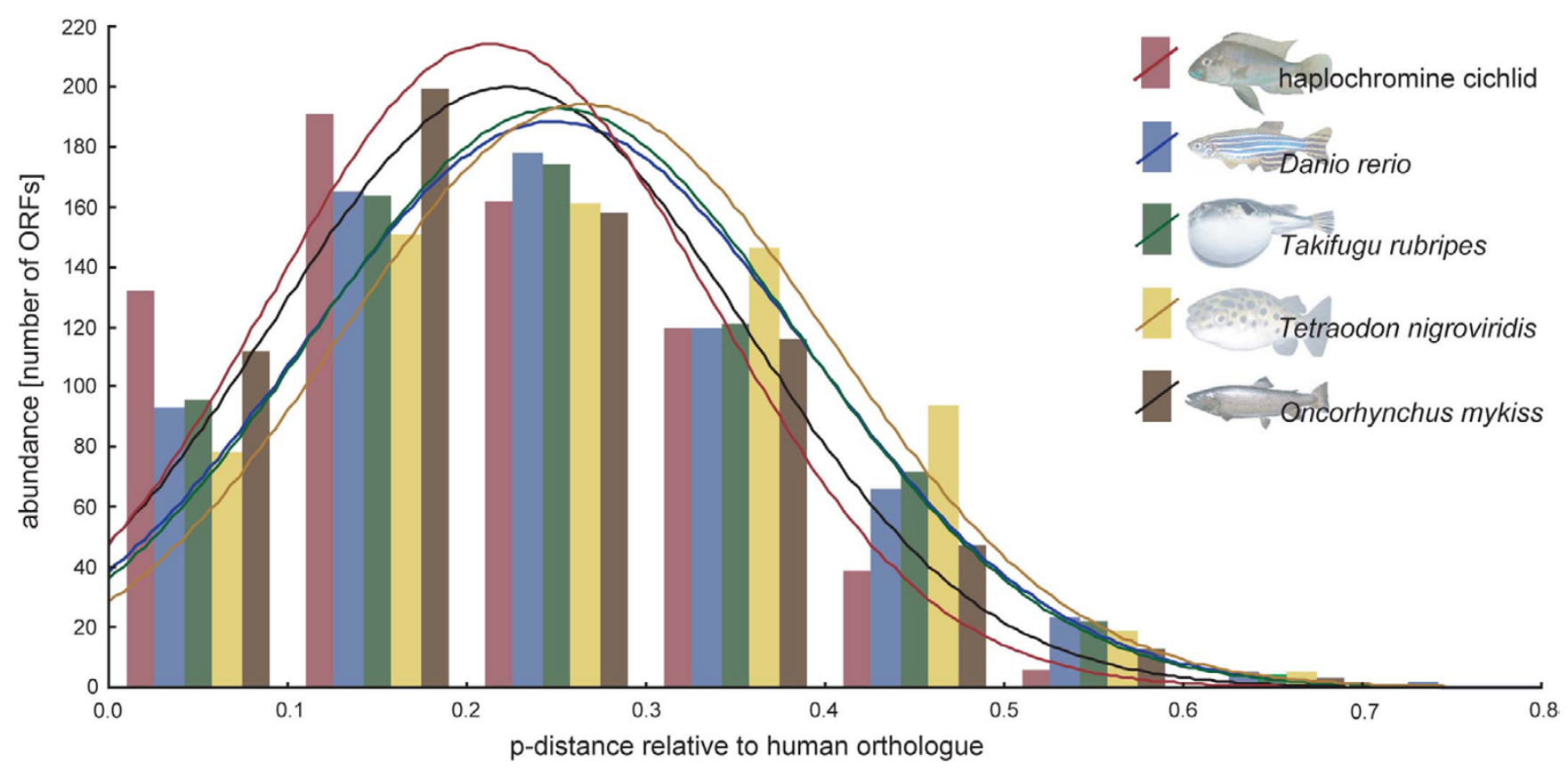

Figure 3

Histogram of the abundance of amino acid sequence divergences of all five fish species (haplochromine cichlid, Danio rerio, Takifugu rubripes, Tetraodon nigroviridis, and Oncorhynchus mykiss) with respect to human genes. $\mathrm{P}$ distances have been calculated for a set of 759 ORFs found in all five fish species and plotted in categories of 0.1 .

We also used the 482 redundant sequences that were found in all three large haplochromine cichlid EST datasets (P. chilotes and P. sp. "redtail sheller" [30]; Astatotilapia burtoni, this study) to calculate mean pairwise p-distances. Within these three cichlid species, we found a mean p-distance of 0.14 between $A$. burtoni and P. chilotes, 0.17 between A. burtoni and P. sp. "redtail sheller", and 0.08 between the two Lake Victoria species P. chilotes and P. sp. "redtail sheller".

We then calculated $\mathrm{K}_{\mathrm{a}} / \mathrm{K}_{\mathrm{s}}$ ratios for all genes with a higher or slower rate of base substitution in cichlids. $\mathrm{K}_{\mathrm{a}} / \mathrm{K}_{\mathrm{s}}$ ratios greater than one, which are indicative of positive selection in that gene, were found in four genes that evolve more slowly in cichlids compared to the other fish species. The highest $\mathrm{K}_{\mathrm{a}} / \mathrm{K}_{\mathrm{s}}$ ratio (3.77) was found in the neuroendocrine convertase subtilisin/kexin type 1 that is responsible for processing large precursor proteins into mature peptide hormones $[55,56]$. In claudin 3 , a member of the claudin family involved in the formation of tight junctions in various tissues [57], the $\mathrm{K}_{\mathrm{a}} / \mathrm{K}_{\mathrm{s}}$ ratio was $1.55 . \mathrm{A} \mathrm{K}_{\mathrm{a}} / \mathrm{K}_{\mathrm{s}}$ ratio of 1.30 was observed in the catalyzing enzyme glutathione peroxidase 3, and a ratio of 1.19 was found in ménage a trois 1 (MNAT1), which is a member of the CDK7-cyclin H complex that functions in cell cycle progression [58], basal transcription, and DNA repair.

\section{Discussion}

Expressed sequence tags are important genomic resources and their numbers in public databases such as GenBank are rapidly increasing. Full-length cDNA and EST sequencing projects typically accompany genome sequencing projects, as these data are essential for the recognition and annotation of genes, the characterization of the transcriptome, the identification of intron-exon boundaries and the detection of splice variants in eukaryotes, etc.[33,34,59-61]. In addition, the standardized procedure of cDNA library construction and normalization, and the comparably low costs of large-scale DNA sequencing facilitate EST projects in organisms for which the whole genome sequencing has not (yet) been completed. Thus, EST sequencing projects outnumber genomesequencing projects - particularly in groups with larger genome sizes such as plants and vertebrates - leading to a large body of sequence data available for comparative analyses. Large-scale EST analyses have been used in many other contexts, such as primary gene expression assays $[62,63]$, the estimation of the total number of genes in an organism [64], cDNA microarray annotations [65], or the construction of genetic linkage maps [66-68]. Expressed sequence tags can further be used for phylogenomics $[36,69]$, and for the identification of microRNAs [70]. 
Despite their many advantages, there are also some problems associated with ESTs. For example, EST sequences typically cover only parts of a gene, so that two sequences of the same gene might not necessarily overlap. That only fragments of a gene are available also leads to problems with homology-based analyses such as BLAST. Then, EST sequences often contain the untranslated regions (UTRs) that are present in mRNAs but do not translate into amino acids. Finally, it is often difficult to figure out the proper reading frame, particularly in shorter ESTs, which impedes certain analyses. A combination of multiple EST projects (as we have done here) helps to alleviate some of the shortcomings inherent in EST data.

We have sequenced, annotated and conducted evolutionary analysis of ESTs of haplochromine cichlids for several reasons. First, this large set of sequence data for cichlid ORFs provides insight into the genome of a representative of haplochromine cichlids, which are a main model system for the study of adaptive evolution and explosive speciation [1-3]. Second, we wanted to extend the existing genomic resources for Astatotilapia burtoni such as a genomic BAC library [27] by establishing cDNA libraries from different tissues. Furthermore, these cDNA libraries provide the basis for annotated cDNA microarrays that are being used for expression analyses in a variety of cichlid species $[22,28,71]$. Finally, we were interested in identifying genes with a different evolutionary rate in the rapidly radiating cichlid lineage compared to other fish species, as well as in identifying genes that show the signature of adaptive evolution in cichlids.

Of the two A. burtoni cDNA libraries that were used for EST sequencing, the normalized mixed tissue library ('pinky') was of better quality. Not only were there much fewer redundant sequences as compared to the brain library, which was mainly due to the normalization step, but also the average insert size was larger and the average read length was longer. Altogether, about $85 \%$ of the sequenced cDNA clones led to high-quality ESTs of a length of $>200$ bp ( $86 \%$ in pinky, and $85 \%$ in brain). In the BLAST searches against Takifugu rubripes, Tetraodon nigroviridis, and Danio rerio, between 14\% (when compared to T. rubripes; e-value $\leq 10^{-50}$ ) and $43 \%$ (when compared to $D$. rerio; e-value $\leq 10^{-5}$ ) of the A. burtoni ESTs led to hits (Fig. 1). This lies well within the range of other EST sequencing projects $[63,65,72]$.

About 8,600 A. burtoni ORFs (or 75\% of the high quality ESTs) were longer than $400 \mathrm{bp}$, and about 3,000 sequences could unambiguously be annotated and classified following the vocabulary provided by the Gene Ontology Consortium [Additional files 1, 2, 3, 4, 5, 6]. According to the Gene Ontology classification, it appears that a broad range of genes involved in functions, proc- esses and compartments are represented in our EST set. This cichlid specific GO slim offers several advantages. First, it offers a rapid visual interpretation of gene subsets. Second, because the cichlid specific slim is built from those sequences used to build a cDNA microarray, it offers maximal power when testing for over- or under-representation of gene lists while reducing the need for correction for multiple hypothesis testing. Finally, it allows for a less experimenter-biased interpretation of microarray results, or other genomics analyses in a manner that can be easily compared between experiments.

One of our main goals was to characterize genes in haplochromine cichlids that show a faster or slower rate of base substitutions in cichlids compared to other fish species, as this is indicative of a relaxed or reinforced selection regime, respectively [35]. To this end, we combined our newly generated ESTs with previously published sequences for Lake Victoria haplochromine cichlids [30] and about 1,000 sequences obtained from a Metriaclima zebra skin cDNA library, which resulted in a total of about 45,000 ORFs. By means of homology searches against human, the two pufferfishes, trout, and zebrafish using local BLAST, we identified a set of 759 ORFs that are present in all species and that show a sufficient degree of homology (e-value $\leq 10^{-50}$ ) for further analyses with EverEST [37]. The number of genes with a cichlid-specific faster or slower rate of molecular evolution (always with human as outgroup) varied when different fish taxa were used in addition to the cichlid ORFs. However, we found a set of 170 genes (48 "faster" and 122 "slower"; Additional files 7,8 ) that appeared in all comparisons and are, thus, good candidates for playing an important role in the evolution of (haplochromine) cichlid fishes.

When characterizing these genes further, by means of calculating $\mathrm{K}_{\mathrm{a}} / \mathrm{K}_{\mathrm{s}}$ ratios, we found that four genes (or $2.35 \%$ of all deviating genes) showed the signature of adaptive evolution in the haplochromine lineage. The highest $\mathrm{K}_{\mathrm{a}} /$ $\mathrm{K}_{\mathrm{s}}$ ratio (3.77) was found in the neuroendocrine convertase subtilisin/kexin type 1, followed by claudin 3, (1.55), glutathione peroxidase 3 (1.50), and ménage a trois 1 (1.19). All gene fragments that show $a K_{a} / K_{s}>1$ are found among the more slowly evolving genes. These genes are now candidate genes for further investigations. The gene with the highest $\mathrm{K}_{\mathrm{a}} / \mathrm{K}_{\mathrm{s}}$ ratio appears particularly interesting. It is known that neuroendocrine factors, such as gonadotropin releasing hormone $(\mathrm{GnRH})$, are involved in regulation of reproduction and behavior in A. burtoni $[56,73]$.

In order to generate hypotheses regarding possible mechanisms by which the rapidly or slowly evolving cichlid genes might contribute to the process of adaptive radiation, we made use of the GO term annotations and cichlid specific slim. Over- and under-represented terms were 
identified among the annotations for the rapidly and slowly evolving cichlid genes (Table 2). Among the 759 ORFs for which p-distances were calculated, over 6,000 total annotations were applied to 647,675 , and 619 ORFs according to biological process, molecular function, and cellular component respectively. Therefore the majority of the 122 slowly evolving and 48 rapidly evolving genes could be classified bioinformatically.

Table 2: Gene Ontology terms which are over- or under-represented among the rapidly or slowly evolving cichlid ORFs. Hypergeometic p-values are reported uncorrected for multiple testing. The number of ORFs of deviating evolutionary rate (\#) relative to the number of core set ORFs (total) is given.

\begin{tabular}{|c|c|c|c|c|c|}
\hline Representation & GO-ID & p-value & \# & total & Description \\
\hline & biological process & \multicolumn{4}{|c|}{42 with higher p-distance (647 annotated) } \\
\hline over & none & & & & \\
\hline \multirow[t]{5}{*}{ under } & GO:0050896 & 0.0161 & 1 & 86 & response to stimulus \\
\hline & GO:0009987 & 0.0439 & 12 & 273 & cellular process \\
\hline & molecular function & \multicolumn{4}{|c|}{44 with higher p-distance ( 675 annotated) } \\
\hline & none & & & & \\
\hline & Cellular component & \multicolumn{4}{|c|}{40 with higher p-distance (6 19 annotated) } \\
\hline over & GO:0015629 & 0.0327 & 6 & 39 & actin cytoskeleton \\
\hline \multirow[t]{2}{*}{ under } & none & & & & \\
\hline & biological process & \multicolumn{4}{|c|}{ I 03 with lower p-distance (647 annotated) } \\
\hline over & GO:0009987 & 0.0024 & 57 & 273 & cellular process \\
\hline over & GO:0007243 & 0.0052 & 8 & 19 & protein kinase cascade \\
\hline over & GO:0007I55 & 0.0205 & 7 & 19 & cell adhesion \\
\hline over & GO:0040007 & 0.0208 & 6 & 15 & growth \\
\hline over & GO:0007I54 & 0.0230 & 25 & 109 & cell communication \\
\hline over & GO:0007267 & 0.0071 & 7 & 16 & cell-cell signaling \\
\hline over & GO:0016477 & 0.0290 & 5 & 12 & cell migration \\
\hline over & GO:0040008 & 0.0290 & 5 & 12 & regulation of growth \\
\hline over & GO:0007409 & 0.0308 & 3 & 5 & axonogenesis \\
\hline over & GO:0007610 & 0.0308 & 3 & 5 & behavior \\
\hline over & GO:0015674 & 0.0308 & 3 & 5 & di-, tri-valent inorganic cation transport \\
\hline over & GO:0019752 & 0.0376 & 10 & 35 & carboxylic acid metabolic process \\
\hline over & GO:0007067 & 0.0402 & 4 & 9 & mitosis \\
\hline over & GO:00074I7 & 0.0402 & 4 & 9 & central nervous system development \\
\hline under & GO:0008I52 & 0.0016 & 63 & 477 & metabolic process \\
\hline under & GO:0046907 & 0.0180 & 2 & 44 & intracellular transport \\
\hline under & GO:0045045 & 0.0295 & 0 & 20 & secretory pathway \\
\hline \multirow[t]{2}{*}{ under } & GO:0009117 & 0.0421 & 0 & 18 & nucleotide metabolic process \\
\hline & molecular function & \multicolumn{4}{|c|}{ I 10 with lower p-distance (675 annotated) } \\
\hline over & GO:0004930 & 0.0157 & 4 & 7 & G-protein \\
\hline over & GO:0003774 & 0.0233 & 6 & 15 & motor activity \\
\hline over & GO:0005262 & 0.0264 & 2 & 2 & calcium channel \\
\hline over & GO:0008047 & 0.0324 & 6 & 16 & enzyme activator activity \\
\hline over & GO:0005509 & 0.0333 & 12 & 43 & calcium ion binding \\
\hline over & GO:0019899 & 0.0435 & 4 & 9 & enzyme binding \\
\hline under & GO:0005525 & 0.0116 & I & 36 & GTP binding \\
\hline under & GO:0005198 & 0.0407 & 8 & 85 & structural molecule activity \\
\hline under & GO:005I082 & 0.0467 & 0 & 17 & unfolded protein binding \\
\hline under & GO:0003743 & 0.0467 & 0 & 17 & translation initiation factor activity \\
\hline under & GO:0003924 & 0.0481 & 1 & 27 & GTPase activity \\
\hline \multirow[t]{2}{*}{ under } & GO:0003676 & 0.0483 & 17 & 147 & nucleic acid binding \\
\hline & cellular component & \multicolumn{4}{|c|}{97 with lower p-distance (619 annotated) } \\
\hline over & GO:001602I & 0.0096 & 22 & 88 & integral to membrane \\
\hline over & GO:0015630 & 0.0388 & 5 & 13 & microtubule cytoskeleton \\
\hline over & GO:0005625 & 0.0479 & 6 & 18 & soluble fraction \\
\hline over & GO:0005615 & 0.0479 & 6 & 18 & extracellular space \\
\hline under & GO:0032991 & 0.0001 & 19 & 222 & macromolecular complex \\
\hline under & GO:0043234 & 0.0015 & 18 & 195 & protein complex \\
\hline under & GO:0043226 & 0.0089 & 56 & 425 & organelle \\
\hline under & GO:0030529 & 0.0139 & 4 & 65 & ribonucleoprotein complex \\
\hline under & GO:0005829 & 0.0267 & 3 & 51 & cytosol \\
\hline under & GO:0005739 & 0.0311 & 6 & 75 & mitochondrion \\
\hline
\end{tabular}


There was a relatively even distribution of rapidly evolving genes across all GO categories. Only three terms, "response to stimulus", "cellular process" and "actin cytoskeleton" deviated significantly from the distribution expected by chance alone. The most significant disproportionate under-representation for the rapidly evolving genes was the category of response to stimulus for which only 1 of the 86 possible annotated ORFs was included on the list.

The distribution across GO categories was highly non-uniform for the slowly evolving genes. Many categories from each ontology were represented by significantly more or fewer ORFs than would be expected by chance. Among those terms over-represented we found several relating to cellular processes such as protein kinase cascade, mitosis, and cell signaling as well as growth and cell adhesion, while metabolic process was under-represented along with the secretory pathway category.

The GO analysis highlights the possible categories of genes that may play an important role in the evolution of the haplochromine cichlid fishes. This analysis presents hypotheses to be tested through focused experimental or sequence analysis. An interesting contrast in GO analysis results was observed between the rapidly evolving genes that showed little tendency to derive from a particular class and slowly evolving genes that were more structured in their distribution. The lack of structure to the distribution of rapidly evolving genes may reflect the possibility that specialization among cichlids occurs along diverse biological pathways rather than a repeated divergence of a given biological process or molecular function. The GO categories that are over-represented among slowly evolving genes could represent genes whose functions are important for phenotypic plasticity or other traits linked to the successful adaptive radiation, while those categories that are under-represented by slowly evolving genes represent categories that are not as tightly constrained.

Our p-distance comparisons between the five fish species and human (as outgroup) also revealed that cichlids show the lowest average p-distance compared to Homo sapiens (Fig. 3). This might be an artifact that is due to the use of the haplochromine cichlid sequence as query for all BLAST searches. Alternatively, as we also found 122 slowly evolving genes in haplochromine cichlids, there might be a tendency in haplochromines to retain ancestral forms and functions. The pairwise average p-distance comparisons between the three cichlid species Paralabidochromis chilotes, Ptyochromis sp. "redtail sheller", and Astatotilapia burtoni revealed that the coalescence time between the two Lake Victoria species $(0.08)$ is about half compared to their coalescence time with A. burtoni $(0.14$ and 0.17 , respectively), which is in concordance to the phylogenetic relationships between these three taxa [4].

\section{Conclusion}

Here we report the sequencing and annotation of more than 11,000 ESTs from the East African haplochromine cichlid Astatotilapia burtoni. Our EST set comprises a broad range of genes involved in functions, processes and compartments. By combining the A. burtoni ESTs with publicly available ORFs from two Lake Victoria haplochromines and subsequent comparisons to other fish model systems, we identify a set of 170 genes with haplochromine-specific differences in evolutionary rates. These genes appear as good candidates for playing an important role in the evolution of the exceptional diversity found in (haplochromine) cichlids. Interestingly, genes that were more slowly evolving in the cichlid lineage were not evenly distributed across Gene Ontology categories; classes that are over-represented could represent genes whose functions are important for successful adaptive radiation. We also identify four genes with a $\mathrm{K}_{\mathrm{a}} / \mathrm{K}_{\mathrm{s}}$ ratio greater than one, which are, hence, likely to have undergone positive selection in haplochromines. The A. burtoni ESTs provide novel insights into the genome of haplochromine cichlids and will serve as valuable resource for researchers working in the field of (cichlid) evolutionary genomics, particularly in the light of the forthcoming sequencing of four cichlid genomes.

\section{Methods \\ Fishes}

Astatotilapia burtoni were kept at Stanford, and at the Tierforschungsanlage of the University of Konstanz under standard conditions (12 h light, $12 \mathrm{~h}$ dark; $26^{\circ} \mathrm{C}$ ). For RNA isolation, fishes were sacrificed after anesthetization with MS 222 (Sigma).

\section{Pinky cDNA Library Construction}

For the preparation of the pinky cDNA library, total RNA was isolated from the following tissues of adult A. burtoni: brain, caudal fin, anal fin (male), lips, muscle, ovary (female), and skin. Additionally, we isolated total RNA from a juvenile individual (about 30 days after fertilization). Total RNA was isolated by guanidine thiocyanate/ phenol-chlorophorm-isoamyl alcohol extraction and lithium-chloride precipitation. The different RNA samples were pooled and cDNA was synthesized using the SMART PCR CDNA Synthesis Kit (Clontech) following the manufacturer's protocol. Amplified cDNA was purified using the QIAquick PCR Purification Kit (Qiagen) and concentrated by ethanol precipitation. The pellet was dissolved in $10 \mu \mathrm{H}_{2} \mathrm{O}$. For normalization, three microliters of purified cDNA were mixed with $1 \mu$ l hybridization buffer (200 $\mathrm{mM}$ HEPES-HCl, pH 8.0; $2 \mathrm{M} \mathrm{NaCl}$ ) and incubated at $95^{\circ} \mathrm{C}$ for 5 minutes and at $70^{\circ} \mathrm{C}$ overnight. Then, $1 \mu \mathrm{l}$ of 
DNAse buffer (500 mM Tris-HCl, $\mathrm{pH} 8.0 ; 50 \mathrm{mM} \mathrm{MgCl}_{2}$ $10 \mathrm{mM}$ DTT) and $0.5 \mu \mathrm{l}$ of DSN enzyme (duplex-specific nuclease; Evrogen, Russia) were added, and the mix was incubated at $65^{\circ} \mathrm{C}$ for 20 minutes. The normalization reaction was terminated by adding $1 \mu \mathrm{l} 50 \mathrm{mM}$ EDTA and incubation at $95^{\circ} \mathrm{C}$ for 7 minutes. Normalized cDNA was PCR amplified (20 cycles) and cloned into PAL 16 vectors.

\section{Brain cDNA Library Construction}

A full-length, directional (EcoRI - XhoI) cDNA library was constructed in Lambda ZapII phage vector (Stratagene) with mRNA from A. burtoni brains (both sexes at all stages of development and social condition were included). Construction of this library has previously been described in [22]. For cDNA sequencing, we used $2 \mu \mathrm{l}$ of purified PCR products, which were also used for the construction of a cDNA microarray [22].

\section{DNA-sequencing and Sequence Analysis}

For sequencing of the normalized pinky cDNA library we used purified plasmid DNA from $1 \mathrm{ml}$ colonies that were grown overnight. Plasmid DNA was directly sequenced using T7 primers and the BigDye Termination Reaction Kit v3.0 (Applied Biosystems) on ABI 3730 and ABI 3100 automated capillary DNA sequencers (Applied Biosystems). Sequences of the brain cDNA library were determined on an ABI 3100 DNA sequencer after cycle sequencing reactions from purified PCR products that were available from the construction of a cDNA microarray [22] using the primer CSVP3 (5'-AAGCGCGCAATTAACCCTCACTA-3') and the BigDye Termination Reaction Kit v3.0 (Applied Biosystems).

Base-calling and quality trimming were performed with phred [74] using a quality score $>20$. Vectors were trimmed with Sequencher 4.2.2 (Genecodes). Those ESTs having a total length of $>200$ bp after quality and vector trimming were considered "high-quality ESTs". Screens for possible contaminations were conducted by blastn searches against the E. coli genome, and the EST_human, EST_mouse and EST_others databases (downloaded in March 2005). Sequences have been deposited in GenBank under accession numbers $\underline{\mathrm{CN} 468542}$ - $\underline{\mathrm{CN} 472211}$ (brain library) and DY625779 - DY632420 (pinky library).

\section{Annotation of A. burtoni ESTs}

High quality A. burtoni ESTs were screened by tblastx searches against protein data from Danio rerio (Zebrafish Sequencing Group at the Sanger Institute), Homo sapiens (GenBank) and Takifugu rubripes (JGI Fugu v3.0) as well as ESTs from Oncorhynchus mykiss and Tetraodon nigroviridis (GenBank) using the standard vertebrate code for translation into amino acids. The expected value thresholds (evalues) were set to $<1 \times 10^{-5},<1 \times 10^{-15}$, and $<1 \times 10^{-50}$. The proper open reading frame for A. burtoni ESTs was determined with EverEST [37], based on the results from these BLAST searches.

For functional annotation of A. burtoni ESTs, we followed the vocabulary provided by the Gene Ontology Consortium using the GO database [75]. Gene Ontology terms were applied to the cichlid assembled sequences by BLAST comparison to the Gene Ontology database (release 200704), which represents protein sequence for all contributed genes for which at least one GO annotation has been applied based on experimental evidence rather than only inferred electronic annotation of sequence. All GO annotations at any confidence level were then transferred from the single best-hit gene using e-value $<10^{-12}$ as a threshold. The collection of GO terms used was "slimmed" in order to produce useful summaries of the annotations.

This cichlid specific slim [Additional files 4, 5, 6] is based upon statistical consideration for analysis of microarray results. The leaf most nodes have been selected for which 20 or more A. burtoni assembled sequences were annotated with this term. Parent nodes were retained only when an additional $20 \mathrm{~A}$. burtoni assembled sequences were included. To assess the enrichment of particular classes of genes among the genes showing deviating rate of molecular evolution, Gene Ontology annotation terms were tested for significant over- and under-representation in either the higher or lower p-distance list using a hypergeometric test implemented in the BINGO plugin [76] for Cytoscape [77]. Due to the exploratory nature of this analysis and controversial application of correction techniques [78], reported p-values are not corrected for multiple testing. Only the representation for the leaf most node is reported except in cases when a larger, parent node showed increased significance. The directed acyclic graphs (DAGs) were created using hierarchical visualization in Cytoscape and manually adjusted to facilitate comprehension.

\section{Evolutionary Analyses}

For evolutionary analyses of ESTs from haplochromine cichlids, we combined our newly generated high-quality ESTs from A. burtoni with previously published ESTs from Paralabidochromis chilotes and Ptyochromis sp. "redtail sheller" [30] and with about 1,000 ESTs obtained from a cDNA library made from Metriaclima zebra skin tissue (W. Salzburger, H. A. Hofmann \& A. Meyer, unpublished). The combined dataset, including more than 45,000 ESTs, was BLASTed against protein data from Danio rerio, Homo sapiens and Takifugu rubripes as well as ESTs from Oncorhynchus mykiss and Tetraodon nigroviridis (see above for source of data) using the translated BLAST routine and the standard vertebrate code. This was done to identify a set of ORFs present in all datasets under study. BLAST searches 
were performed with an e-value of $<1 \times 10^{-50}$ in order to achieve high levels of confidence in the similarity searches. The cichlid query sequences and the best hits from every single BLAST search against the different databases were imported into EverEST [37].

In order to identify coding sequences showing a deviating rate of molecular evolution in haplochromine cichlids compared to other fish lineages we applied the triangle method implemented in EverEST. In this approach, the query sequences are aligned to their best BLAST hits in two ingroup and one outgroup taxa using the T-Coffee algorithm [79] as implemented in EverEST [37]. This reveals multiple sequence alignments consisting of four taxa. Then, uncorrected pairwise p-distances are calculated for all taxon pairs in each alignment, which are used to construct neighbor-joining trees and, after rooting with the outgroup sequences, for a global ternary representation. A relative rate test was applied to each of the orthologous groups. We applied the nonparametric rate test developed by Tajima [80], and compared the genes with their human and their fish orthologs in order to identify higher or lower substitution rates.

For these analyses, we used the human sequences as outgroup since tetrapods are valid outgroup taxa for teleost fish and the human genome is the most complete and best annotated genome among those. In addition to our haplochromine cichlid query sequences, we used different sets of ingroup taxa in order to minimize biasing effects due to sparse taxon sampling. We used the following combinations of taxa for our evolutionary rate analyses using 759 ORFs that have been found in all datasets: (human, (haplochromine cichlid, Danio rerio, Takifugu rubripes)) (Fig. 2a), (human, (haplochromine cichlid, Danio rerio, Tetraodon nigroviridis)) (not shown), (human, (haplochromine cichlid, Danio rerio, Oncorhynchus mykiss)) (not shown). As a control, we also analyzed a data set without the cichlid-query sequences for the same set of ORFs (human, (Danio rerio, Oncorhynchus mykiss, Takifugu rubripes)) (Fig. 2b). We note that this approach might lead to an underestimation of the number of faster evolving genes, as genes that accumulated too many mutations are likely not to be chosen in the stringent initial BLAST searches. We would also like to point out that some of the observed rate differences might have accumulated on the evolutionary lineage leading to the cichlids but before the cichlids have evolved as a group.

For orthologous groups, where the p-distance in the haplochromine cichlids were significantly $(\mathrm{p}<0.05)$ higher or lower compared to other fish, the ratio of the number of nonsynonymous substitutions per nonsynonymous site $\left(\mathrm{K}_{\mathrm{a}}\right)$ to the number of synonymous substitutions per synonymous site $\left(\mathrm{K}_{\mathrm{s}}\right)$ was calculated based on a likeli- hood approach [81] to evaluate the selective forces acting on those proteins. The $\mathrm{K}_{\mathrm{a}} / \mathrm{K}_{\mathrm{s}}$ ratio is an indicator of the form of sequence evolution, with $\mathrm{K}_{\mathrm{a}} / \mathrm{K}_{\mathrm{s}}>>1$ providing strong evidence that positive selection has acted to change the protein sequence.

We also constructed a histogram of amino acid sequence divergence of all five fish datasets with respect to homologous human sequences. We finally used the redundant sequences in the three datasets $P$. chilotes, $P$. sp. "redtail sheller", and A. burtoni to calculate pairwise average p-distances.

\section{Abbreviations}

DAG, directed acyclic graph; EST, expressed sequence tag; $\mathrm{GO}$, gene ontology; ORF, open reading frame

\section{Authors' contributions}

WS, HAH and AM designed the study. WS and HAH were involved in library construction; WS and IB carried out the molecular work; WS, DS, SCPR, and IB performed the analyses. All authors contributed to the preparation of the manuscript. They read and approved the final version.

\section{Additional material}

\section{Additional file 1}

Gene ontology table (generic GO slim subset for molecular function). Hierarchical classification of the GO slim subset for molecular function. Indented terms are children of parent terms listed above. For each term, the number of A. burtoni assembled sequences that match genes to which Gene Ontology annotations have been assigned at, or below, this general level is given. Note that genes may be assigned to more than one term and child terms may have more than one parent term. For parent terms, the total number of A. burtoni assembled sequences is given in parentheses. Match means that the annotation derives from a gene that was the "best hit" for the A. burtoni sequence at and e-value $<10^{-12}$.

Click here for file

[http://www.biomedcentral.com/content/supplementary/14712164-9-96-S1.PDF]

\section{Additional file 2}

Gene ontology table (generic GO slim subset for biological process). Hierarchical classification of the GO slim subset for biological process. Indented terms are children of parent terms listed above. Genes may be assigned to more than one term. For each term, the number of A. burtoni assembled sequences that match genes to which Gene Ontology annotations have been assigned at, or below, this general level is given. Note that genes may be assigned to more than one term and child terms may have more than one parent term. For parent terms, the total number of A. burtoni assembled sequences is given in parentheses. Match means that the annotation derives from a gene that was the "best hit" for the A. burtoni sequence at and e-value $<10^{-12}$.

Click here for file

[http://www.biomedcentral.com/content/supplementary/14712164-9-96-S2.PDF] 


\section{Additional file 3}

Gene ontology table (generic GO slim subset for cellular component). Hierarchical classification of the GO slim subset for cellular component. Indented terms are children of parent terms listed above. Genes may be assigned to more than one term. For each term, the number of A. burton assembled sequences that match genes to which Gene Ontology annotations have been assigned at, or below, this general level is given. Note that genes may be assigned to more than one term and child terms may have more than one parent term. For parent terms, the total number of A. burtoni assembled sequences is given in parentheses. Match means that the annotation derives from a gene that was the "best hit" for the A. burtoni sequence at and e-value $<10^{-12}$.

Click here for file

[http://www.biomedcentral.com/content/supplementary/14712164-9-96-S3.PDF]

\section{Additional file 4}

Directed acyclic graph (DAG) of the cichlid specific Gene ontology (GO) slim for molecular function. The graph shows the cichlid specific GO slim for molecular function. Molecular function terms were selected for inclusion in the ontologies such that leaf nodes include approximately 20 annotated genes. Circle size represents relative number of genes annotated to each parent node.

Click here for file

[http://www.biomedcentral.com/content/supplementary/14712164-9-96-S4.JPEG]

\section{Additional file 5}

Directed acyclic graph (DAG) of the cichlid specific Gene ontology (GO) slim for biological process. The graph shows the cichlid specific GO slim for biological process. Biological process terms were selected for inclusion in the ontologies such that leaf nodes include approximately 20 annotated genes. Circle size represents relative number of genes annotated to each parent node.

Click here for file

[http://www.biomedcentral.com/content/supplementary/14712164-9-96-S5.JPEG]

\section{Additional file 6}

Directed acyclic graph (DAG) of the cichlid specific Gene ontology (GO) slim for cellular component. The graph shows the cichlid specific GO slim for cellular component. Cellular component terms were selected for inclusion in the ontologies such that leaf nodes include approximately 20 annotated genes. Circle size represents relative number of genes annotated to each parent node.

Click here for file

[http://www.biomedcentral.com/content/supplementary/1471-

2164-9-96-S6.JPEG]

\section{Additional file 7}

ESTs with higher p-distances. The table shows ESTs where the p-distance between Homo sapiens and haplochromine cichlid amino acid sequences is significantly higher as compared to other fish species (Danio rerio, Takifugu rubripes, Tetraodon nigroviridis and Oncorhynchus mykiss). Annotation means that the Homo sapiens gene was "best hit" for the cichlid sequence (and e-value $<10^{-50}$ ).

Click here for file

[http://www.biomedcentral.com/content/supplementary/14712164-9-96-S7.PDF]

\section{Additional file 8}

ESTs with smaller p-distances. The table shows ESTs where the p-distance between Homo sapiens and haplochromine cichlid amino acid sequences is significantly smaller as compared to other fish species (Danio rerio, Takifugu rubripes, Tetraodon nigroviridis, and Oncorhynchus mykiss). Annotation means that the Homo sapiens gene was "best hit" for the Cichlid sequence (and e-value $<10^{-50}$ ).

Click here for file

[http://www.biomedcentral.com/content/supplementary/14712164-9-96-S8.PDF]

\section{Acknowledgements}

We thank E. Hespeler for technical assistance in the laboratory, P. Jantzen for assistance with GO figures and tables, and R. D. Fernald, in whose laboratory the brain cDNA library was constructed; WS was supported by a Marie Curie Fellowship of the EU, and grants from the LandesstiftungBaden Württemberg gGmbH and the Center for Junior Research Fellows, University of Konstanz; SCPR was supported by an NIH-NRSA grant; $\mathrm{HAH}$ was supported by a NIH-NIGMS grant GM068763, the Bauer Center for Genomics Research at Harvard University and the Institute for Cellular and Molecular Biology at the University of Texas, Austin; AM was supported by the Deutsche Forschungsgemeinschaft (DFG) and the University of Konstanz.

\section{References}

I. Kocher TD: Adaptive evolution and explosive speciation: the cichlid fish model. Nature Reviews Genetics 2004, 5:288-298.

2. Salzburger W, Meyer A: The species flocks of East African cichlid fishes: recent advances in molecular phylogenetics and population genetics. Naturwissenschaften 2004, 9 I:277-290.

3. Kornfield I, Smith PF: African Cichlid Fishes: Model systems for evolutionary biology. Annu Rev Ecol Syst 2000, 3 I : 163-196.

4. Salzburger W, Mack T, Verheyen E, Meyer A: Out of Tanganyika: Genesis, explosive speciation, key-innovations and phylogeography of the haplochromine cichlid fishes. BMC Evolutionary Biology 2005, 5: I7.

5. Verheyen E, Salzburger W, Snoeks J, Meyer A: Origin of the superflock of cichlid fishes from Lake Victoria, East Africa. Science 2003, 300:325-329.

6. Genner MJ, Seehausen O, Lunt DH, Joyce DA, Shaw PW, Carvalho GR, Turner GF: Age of cichlids: new dates for ancient lake fish radiations. Mol Biol Evol 2007, 24:1269-1282.

7. Fryer G, lles TD: The cichlid fishes of the Great Lakes of Africa: Their biology and Evolution. Edinburgh: Oliver \& Boyd; 1972.

8. Barluenga M, Stolting KN, Salzburger W, Muschick M, Meyer A: Sympatric speciation in Nicaraguan crater lake cichlid fish. Nature 2006, 439:719-723.

9. Schliewen UK, Tautz D, Paabo S: Sympatric speciation suggested by monophyly of crater lake cichlids. Nature 1994, 368:629-632.

10. Kocher TD, Conroy JA, McKaye KR, Stauffer JR: Similar morphologies of cichlid fish in lakes Tanganyika and Malawi are due to convergence. Mol Phylogenet Evol 1993, 2:158-165.

II. Stiassny MLj, Meyer A: Cichlids of the Rift Lakes. Scientific American 1999, 280:64-69.

12. Meyer A: Phylogenetic relationships and evolutionary processes in East African cichlids. Trends in Ecology and Evolution 1993, 8:279-284.

13. Liem KF: Evolutionary strategies and morphological innovations: cichlid pharyngeal jaws. Systematic Zoology 1973, 22:425-44I.

14. Crapon de Caprona MD, Fritzsch B: Interspecific fertile hybrids of haplochromine Cichlidae (Teleostei) and their possible importance for speciation. Netherlands Journal of Zoology 1984, 34:503-538.

15. Albertson RC, Kocher TD: Genetic architecture sets limits on transgressive segregation in hybrid cichlid fishes. Evolution Int J Org Evolution 2005, 59:686-690. 
16. Streelman JT, Albertson RC, Kocher TD: Genome mapping of the orange blotch colour pattern in cichlid fishes. Mol Ecol 2003, | 2:2465-247|

17. Albertson RC, Streelman JT, Kocher TD: Directional selection has shaped the oral jaws of Lake Malawi cichlid fishes. Proc Natl Acad Sci USA 2003, 100:5252-5257.

18. Albertson RC, Streelman JT, Kocher TD, Yelick PC: Integration and evolution of the cichlid mandible: the molecular basis of alternate feeding strategies. Proc Natl Acad Sci USA 2005, 102:16287-16292

19. Terai Y, Morikawa N, Okada N: The evolution of the pro-domain of bone morphogenetic protein 4 (Bmp4) in an explosively speciated lineage of East African cichlid fishes. Mol Biol Evol 2002, 19:1628-1632.

20. Sugie A, Terai $Y$, Ota R, Okada N: The evolution of genes for pigmentation in African cichlid fishes. Gene 2004, 343:337-346.

21. Carleton KL, Kocher TD: Cone opsin genes of african cichlid fishes: tuning spectral sensitivity by differential gene expression. Mol Biol Evol 200I, I 8: 1540-I550.

22. Renn SC, Aubin-Horth N, Hofmann HA: Biologically meaningful expression profiling across species using heterologous hybridization to a cDNA microarray. BMC Genomics 2004, 5:42.

23. Lee BY, Lee WJ, Streelman JT, Carleton KL, Howe AE, Hulata G, Slet$\tan$ A, Stern JE, Terai Y, Kocher TD: A Second Generation Genetic Linkage Map of Tilapia (Oreochromis spp.). Genetics 2005, I 70:237-244.

24. Kocher TD, Lee WJ, Sobolewska H, Penman D, McAndrew B: A genetic linkage map of a cichlid fish, the tilapia (Oreochromis niloticus). Genetics 1998, I48:1225-1232

25. Katagiri T, Asakawa S, Minagawa S, Shimizu N, Hirono I, Aoki T: Construction and characterization of BAC libraries for three fish species; rainbow trout, carp and tilapia. Anim Genet 200I, 32:200-204.

26. Watanabe M, Kobayashi N, Fujiyama A, Okada N: Construction of a BAC library for Haplochromis chilotes, a cichlid fish from Lake Victoria. Genes Genet Syst 2003, 78: 103-105.

27. Lang M, Miyake T, Braasch I, Tinnemore D, Siegel N, Salzburger W, Amemiya CT, Meyer A: A BAC library of the East African haplochromine cichlid fish Astatotilapia burtoni. J Exp Zoolog $B$ Mol Dev Evol 2006, 306B:35-44.

28. Kijimoto T, Watanabe M, Fujimura K, Nakazawa M, Murakami $Y$, Kuratani S, Kohara Y, Gojobori T, Okada N: cimpl, a novel actin family metalloproteinase gene from East African cichlids, is differentially expressed between species during growth. Mol Biol Evol 2005, 22:1649-1660.

29. Kobayashi N, Watanabe M, Kijimoto T, Fujimura K, Nakazawa M, Ikeo K, Kohara Y, Gojobori T, Okada N: magp4 gene may contribute to the diversification of cichlid morphs and their speciation. Gene 2006, 373:126-133.

30. Watanabe M, Kobayashi N, Shin-i T, Horiike T, Tateno Y, Kohara Y, Okada N: Extensive analysis of ORF sequences from two different cichlid species in Lake Victoria provides molecular evidence for a recent radiation event of the Victoria species flock: identity of EST sequences between Haplochromis chilotes and Haplochromis sp. "Redtailsheller". Gene 2004, 343:263-269.

31. [http://cichlid.biosci.utexas.edu/html/cichlid genomics.html]

32. [http://www.cichlidgenome.org].

33. Gibson G, Muse SV: A Primer of Genome Science. Sunderland, MA: Sinauer Associates, Inc; 2002.

34. Gerhold D, Caskey CT: It's the genes! EST access to human genome content. Bioessays 1996, I8:973-98I.

35. Steinke D, Salzburger W, Braasch I, Meyer A: Many genes in fish have species-specific asymmetric rates of molecular evolution. BMC Genomics 2006, 7:20.

36. Steinke D, Salzburger W, Meyer A: Higher teleostean relationships revealed from genome-wide phylogenetic analyses. Mol Evol 2006 in press.

37. Steinke D, Salzburger W, Meyer A: EverEST - a phylogenomic EST database approach. PhyloInformatics 2004, 6:1-4

38. Wasmuth JD, Blaxter ML: prot4EST: translating expressed sequence tags from neglected genomes. BMC Bioinformatics 2004, 5: 187.

39. Nilsson RH, Rajashekar B, Larsson KH, Ursing BM: galaxieEST: addressing EST identity through automated phylogenetic analysis. BMC Bioinformatics 2004, 5:87.
40. Wickler W: 'Egg-dummies' as natural releasers in mouthbreeding cichlids. Nature 1962, 194:1092-1093.

4I. Wickler W: Zur Stammesgeschichte funktionell korrelierter Organ- und Verhaltensmerkmale: Ei-Attrappen und Maulbrüten bei afrikanischen Cichliden. Zeitschrift für Tierpsychologie 1962, 19:129-164.

42. Wickler W: Haplochromis burtoni (Cichlidae) Ablaichen. In Encyclopedia Cinematographica Göttingen: Institut für den wissenschaftlichen Film; 1969.

43. Crapon de Caprona MD: Olfactory communication in a cichlid fish, Haplochromis burtoni. Zeitschrift für Tierpsychologie 1980, 52:113-134.

44. Grosenick L, Clement TS, Fernald RD: Fish can infer social rank by observation alone. Nature 2007, 445:429-432.

45. Hofmann HA, Fernald RD: What cichlids tell us about the social regulation of brain and behavior. Journal of Aquariculture and Aquatic Sciences 200I, 9: I-I5.

46. Hofmann HA: Functional genomics of neural and behavioral plasticity. J Neurobiol 2003, 54:272-282.

47. Robison RR, White RB, Illing N, Troskie BE, Morley M, Millar RP, Fernald RD: Gonadotropin-releasing hormone receptor in the teleost Haplochromis burtoni: structure, location, and function. Endocrinology 200I, 142:1737-1743.

48. Kroger RH, Campbell MC, Fernald RD: The development of the crystalline lens is sensitive to visual input in the African cichlid fish, Haplochromis burtoni. Vision Res 200I, 41:549-559.

49. Hagedorn M, Mack AF, Evans B, Fernald RD: The embryogenesis of rod photoreceptors in the teleost fish retina, Haplochromis burtoni. Brain Res Dev Brain Res 1998, 108:217-227.

50. Meyer A, Kocher TD, Basasibwaki P, Wilson AC: Monophyletic origin of Lake Victoria cichlid fishes suggested by mitochondrial DNA sequences. Nature 1990, 347:550-553.

51. Salzburger W, Meyer A, Baric S, Verheyen E, Sturmbauer C: Phylogeny of the Lake Tanganyika cichlid species flock and its relationship to the Central and East African haplochromine cichlid fish faunas. Syst Biol 2002, 5 I: I I 3-135.

52. Consortium TGO: Creating the gene ontology resource: design and implementation. Genome Res 200I, I I:I425-I433.

53. Ashburner M, Ball CA, Blake JA, Botstein D, Butler H, Cherry JM, Davis AP, Dolinski K, Dwight SS, Eppig JT, Harris MA, Hill DP, IsselTarver L, Kasarskis A, Lewis S, Matese JC, Richardson JE, Ringwald M, Rubin GM, Sherlock G: Gene ontology: tool for the unification of biology. The Gene Ontology Consortium. Nat Genet 2000, 25:25-29.

54. [http://www.geneontology.org/GO.slims.shtml]

55. Jansen E, Ayoubi TA, Meulemans SM, Van de Ven WJ: Neuroendocrine-specific expression of the human prohormone convertase I gene. Hormonal regulation of transcription through distinct CAMP response elements. I Biol Chem 1995, 270:1539|-I5397.

56. Hofmann HA: Gonadotropin-releasing hormone signaling in behavioral pasticity. Current Opinion in Neurobiology 2006, 16:343-350

57. Morita K, Furuse M, Fujimoto K, Tsukita S: Claudin multigene family encoding four-transmembrane domain protein components of tight junction strands. Proc Natl Acad Sci USA 1999, 96:5II-5I6.

58. Talukder AH, Mishra SK, Mandal M, Balasenthil S, Mehta S, Sahin AA, Barnes CJ, Kumar R: MTAI interacts with MATI, a cyclindependent kinase-activating kinase complex ring finger factor, and regulates estrogen receptor transactivation functions. J Biol Chem 2003, 278: | |676- I |685.

59. Gupta S, Zink D, Korn B, Vingron M, Haas SA: Strengths and weaknesses of EST-based prediction of tissue-specific alternative splicing. BMC Genomics 2004, 5:72.

60. Banfi S, Borsani G, Rossi E, Bernard L, Guffanti A, Rubboli F, Marchitiello A, Giglio S, Coluccia E, Zollo M, Zuffardi O, Ballabio A: Identification and mapping of human cDNAs homologous to Drosophila mutant genes through EST database searching. Nat Genet 1996, 13:167-174.

61. Bailey LC Jr, Searls DB, Overton GC: Analysis of EST-driven gene annotation in human genomic sequence. Genome Res 1998, 8:362-376.

62. Schmitt AO, Specht T, Beckmann G, Dahl E, Pilarsky CP, Hinzmann $B$, Rosenthal $A$ : Exhaustive mining of EST libraries for genes 
differentially expressed in normal and tumour tissues. Nucleic Acids Res 1999, 27:425I-4260.

63. Habermann B, Bebin AG, Herklotz S, Volkmer M, Eckelt K, Pehlke K, Epperlein HH, Schackert HK, Wiebe G, Tanaka EM: An Ambystoma mexicanum EST sequencing project: analysis of 17,352 expressed sequence tags from embryonic and regenerating blastema cDNA libraries. Genome Biol 2004, 5:R67.

64. Ewing $B$, Green $P$ : Analysis of expressed sequence tags indicates 35,000 human genes. Nat Genet 2000, 25:232-234.

65. Whitfield CW, Band MR, Bonaldo MF, Kumar CG, Liu L, Pardinas JR, Robertson HM, Soares MB, Robinson GE: Annotated expressed sequence tags and cDNA microarrays for studies of brain and behavior in the honey bee. Genome Res 2002, 12:555-566.

66. Smith JJ, Kump DK, Walker JA, Parichy DM, Voss SR: A Comprehensive EST Linkage Map for Tiger Salamander and Mexican Axolotl: Enabling Gene Mapping and Comparative Genomics in Ambystoma. Genetics 2005.

67. Scheetz TE, Raymond MR, Nishimura DY, McClain A, Roberts C, Birkett C, Gardiner J, Zhang J, Butters N, Sun C, Kwitek-Black A, Jacob H, Casavant TL, Soares MB, Sheffield VC: Generation of a highdensity rat EST map. Genome Res 200 I, I I:497-502.

68. Lorenzen MD, Doyungan Z, Savard J, Snow K, Crumly LR, Shippy TD, Stuart J], Brown SJ, Beeman RW: Genetic linkage maps of the red flour beetle, Tribolium castaneum, based on bacterial artificial chromosomes and expressed sequence tags. Genetics 2005, I 70:74I-747.

69. Philippe $H$, Lartillot $N$, Brinkmann $H$ : Multigene analyses of bilaterian animals corroborate the monophyly of Ecdysozoa, Lophotrochozoa, and Protostomia. Mol Biol Evol 2005, 22:1246-1253

70. Zhang BH, Pan XP, Wang QL, Cobb GP, Anderson TA: Identification and characterization of new plant microRNAs using EST analysis. Cell Res 2005, I5:336-360.

7I. Aubin-Horth N, Desjardins JK, Martei YM, Balshine S, Hofmann HA Masculinized dominant females in a cooperatively breeding species. Mol Ecol 2007, 16:1349-1358.

72. Rise ML, von Schalburg KR, Brown GD, Mawer MA, Devlin RH, Kuipers N, Busby M, Beetz-Sargent M, Alberto R, Gibbs AR, Hunt P, Shukin R, Zeznik JA, Nelson C, Jones SR, Smailus DE, Jones SJ, Schein JE, Marra MA, Butterfield YS, Stott JM, Ng SH, Davidson WS, Koop BF: Development and application of a salmonid EST database and cDNA microarray: data mining and interspecific hybridization characteristics. Genome Res 2004, I 4:478-490.

73. Trainor BC, Hofmann HA: Somatostatin regulates aggressive behavior in an African cichlid fish. Endocrinology 2006 147:5 II $9-5125$

74. [http://www.genome.washington.edu/UWGC/analysistools/ Phred.cfm].

75. [http://www.geneontology.org]

76. Maere S, Heymans K, Kuiper M: BiNGO: a Cytoscape plugin to assess overrepresentation of gene ontology categories in biological networks. Bioinformatics 2005, 21:3448-3449.

77. [http://www.cytoscape.org/].

78. Ge YC, Dudoit S, Speet TP: Resampling-based multiple testing for microarray data analysis. Test 2003, I 2: I-77.

79. Notredame C, Higgins DG, Heringa J: T-Coffee: A novel method for fast and accurate multiple sequence alignment. J Mol Biol 2000, 302:205-2I7.

80. Tajima F: Simple methods for testing the molecular evolutionary clock hypothesis. Genetics 1993, I35:599-607.

81. Yang Z: Likelihood ratio tests for detecting positive selection and application to primate lysozyme evolution. Mol Biol Evol 1998, 15:568-573.
Publish with Biomed Central and every scientist can read your work free of charge

"BioMed Central will be the most significant development for disseminating the results of biomedical research in our lifetime. "

Sir Paul Nurse, Cancer Research UK

Your research papers will be:

- available free of charge to the entire biomedical community

- peer reviewed and published immediately upon acceptance

- cited in PubMed and archived on PubMed Central

- yours - you keep the copyright

Submit your manuscript here:

http://www.biomedcentral.com/info/publishing_adv.asp
BiolMedcentral 